\section{IUD/IUS insertions and atropine}

We have read with interest the correspondence relating to intrauterine device (IUD)/intrauterine system (IUS) insertions and atropine. This letter aims to provide an understanding of how this guidance was developed.

The Clinical Standards Committee is responsible for providing guidance on clinical governance in the form of auditable standards to enable the provision of high-quality patient-centred care. There is no division within the Standards between that which should be provided by a doctor or by a nurse, unless there is a clinician-specific issue, for example, issuing medication under a patient group direction (PGD) for non-prescribers.

The updated Resuscitation Standard of the Faculty of Sexual and Reproductive Healthcare was published in November $2010 .{ }^{1}$ Work began on updating the document in January 2010 and during that time any new evidence-based guidance was reviewed and updated. Not only was the issue of intravenous atropine in IUD fitting reviewed but also other guidance with regard to service equipment and safety.

The Resuscitation Council was contacted during the production of the Standard and advised us that there would be no significant changes to their recommendations. Their updated guidelines were published in October $2010^{2}$ and state that the emergency management for bradycardia (heart rate $<60 \mathrm{bpm}$ ) is to "treat any reversible causes" but if adverse signs are present, for example, 'shock' or 'syncope' the recognised treatment is $500 \mu \mathrm{g}$ atropine intravenous, which may be repeated. ${ }^{3}$ We acknowledge that many cases of bradycardia may resolve with basic measures but Point 5.5 of the FSRH Resuscitation Guideline states that: "Significant bradycardia may result from IUD/IUS insertions so whenever this procedure is performed, a clinician competent in intravenous injections should be available in case intravenous atropine is required".

There is no differentiation as to whether this clinician should be a doctor, nurse or paramedic. There is no specification that a doctor has to be present, only that "a clinician competent" is available if the clinician performing the procedure is not competent themselves.

There have been several letters published in this Journal raising concerns, ${ }^{4-8}$ and in addition we have dealtwith queries both during the consultation period and with regard to both the ratified Standard and the original joint statement made with the Associate Members' Working Group (AMWG) on 'The Role of Nurses in Managing Persistent Bradycardia During Intrauterine Contraceptive Insertion by a Nurse in Sexual Health Services'. ${ }^{9}$ We have received many comments about the emergency use of atropine and recognise that the need for emergency use is rare. After discussion with the Resuscitation Council, an additional statement was added to the introduction of the Standard:

"It is important that each service identifies the level of equipment and training needed to deal with common emergencies on the basis of local risk assessment".

This statement would allow services to perform a local risk assessment and identify alternative protocols for dealing 
with emergency procedures if this was felt locally appropriate. For example, it may be more important for a remote rural service to have someone available to administer intravenous atropine than in urban areas where rapid access to emergency services is more likely.

The Resuscitation Standard also highlights the need for regular Basic Life Support and anaphylaxis training, which also are theoretical risks within services, and we would support the view ${ }^{10}$ that while these are also exceptionally rare emergencies, it is generally accepted that these are essential risk management standards.

The Resuscitation Council have agreed to support us when we next update the Standard and the joint statement made with the AMWG, but we will keep the advice under review so that we can incorporate any new evidence as it becomes available.

\section{Jane Dickson}

Vice Chair, FSRH Clinical Standards Committee, London, UK; jane.dickson@nhs.net

\section{Asha Kasliwal}

Chair, FSRH Clinical Standards Committee, London, UK; asha.kasliwal@manchester.nhs.uk

\section{Sarah Randall}

Chair, FSRH Associate Members' Working Group، London, UK

\section{Competing interests None.}

J Fam Plann Reprod Health Care 2011;37:118-119. doi:10.1136/jprhc.2011.0086

\section{REFERENCES}

1 Faculty of Sexual and Reproductive Healthcare. Service Standards for Resuscitation in Sexual Health Services. 2010. http://www.fsrh.org/admin/uploads/ ServiceStandardsResuscitationSHServices.pdf [accessed 24 January 2011].

2 Resuscitation Council (UK). Resuscitation Guidelines 2010. 2010. http://www.resus.org.uk/ pages/guide.htm [accessed 24 January 2011].

3 Resuscitation Council (UK). Adult Bradycardia Algorithm. 2010. http://www.resus.org.uk/pages/ bradalgo.pdf [accessed 24 January 2011].

4 Hollingworth B. Need for IUD fitters to have expertise in resuscitation [Letter]. J Fam Plann Reprod Health Care 2010;36:179-180.

5 Mansour D. Nurse training and the need for IUD fitters to have expertise in resuscitation [Letter]. J Fam Plann Reprod Health Care 2010;36:180.

6 Gormley M Eady A. IUD fitters and training in resuscitation [Letter]. J Fam Plann Reprod Health Care 2010;36:254.

7 Davies K. IUD nurse fitters and resuscitation [Letter]. J Fam Plann Reprod Health Care 2010;36:254

8 Devonald B. IUD fitters and training in resuscitation [Letter]. J Fam Plann Reprod Health Care 2011;37:61.

9 Joint Statement Produced by the Associate Members' Working Group and the Clinical Standards Committee on 'The Role of Nurses in Managing Persistent Bradycardia During Intrauterine Contraceptive Insertion in Sexual
Health Services'. 2009. http://www.fsrh.org/ admin/uploads/491 BradycardiaStatment280709. pdf [accessed 13 February 2011].

10 Gallagher J. IUD/IUS insertion and atropine

[Letter]. J Fam Plann Reprod Health Care

2011:37:61. 